\title{
Drogen und Musik - ein Überblick
}

\section{J. Fachner}

\section{Drugs and Music: an Overview}

\section{Zusammenfassung}

Manche Klienten berichten über drogeninduzierte Wahrnehmungsveränderungen von Musik. In diesem Überblick werden solche Wirkungen beschrieben, der kulturelle Kontext sozialpharmakologisch aufgerissen, Charakteristika von Drogenwirkungen und ihr Einfluss auf die emotionale und kognitive Verarbeitung von Musik dargestellt. Drogeninduzierte Zeit- und Raumwahrnehmungsveränderungen als auch kognitiv-emotionale Bewertungsänderungen lassen temporär andere zerebral vermittelte audiometrische Skalierungen der psychoakustischen Qualität, Melodik, Rhythmik und Intensität der Schallereignisse zu.

\section{Schlüisselwörter}

Musik · Drogengebrauch · Rituale · Kultur · veränderte Bewusstseins- und Wahrnehmungszustände · Sozialpharmakologie · Therapie

\section{Abstract}

Clients often report drug-induced alteration of music perception. This overview of scientific publications aims to describe such alterations, cultural context, some characteristics of drug action and its influence on emotional and cognitive processing of music. Music and drug action are processed in same limbic brain areas. Drug rituals with music served for trading and initiation into codified knowledge legacy, enable access to archetype symbols. Artists often use them creatively to vary their perspective on conditioned perceptive patterns. Social pharmacological aspects allow to distinct combinations of music style and consumer patterns. Psychedelic drugs act on time and space perception, induce changes of cognitive-emotional valence and therefore induce temporarily changed audio metric scales of psycho acoustic qualities, melody, rhythm and intensity of acoustic events. Drug induced cross modal intensification lead to more vivid association and vision correlated to the music used in a guided therapeutic context.

Key words

Music $\cdot$ drug use $\cdot$ rituals $\cdot$ culture $\cdot$ altered states of consciousness and perception $\cdot$ social pharmacology $\cdot$ therapy

\section{Hintergrüinde}

Musik und Rausch scheinen gemeinsame emotionale Verarbeitungsformen zu haben, zumindest was die Verarbeitung im limbischen System betrifft. Wohl jeder bevorzugt eine besondere Musikrichtung, die ihm gefällt. Einzelne Musikstücke daraus können einem sogar einen Schauer („Chills”) über den Rücken laufen lassen und anhand dieser haben Blood und Zatorre zeigen können, dass die musikalische Information auch Gehirnstrukturen erreichen, die an der Vermittlung von Emotionen beteiligt sind. Während der Lieblingsmelodie änderte sich nicht nur die Aktivität des autonomen Nervensystems, wie durch Herzschlag-

Institutsangaben

Institut für Musiktherapie, Fakultät für Medizin, Lehrstuhl für Qualitative Forschung in der Medizin

(Lehrstuhlinhaber: Prof. Dr. phil. D. Aldridge), Universität Witten/Herdecke

Korrespondenzadresse

Dipl. Päd. Dr. Jörg Fachner · Lehrstuhl für Qualitative Forschung in der Medizin, Institut für Musiktherapie, Fakultät für Medizin, Universität Witten/Herdecke · Alfred-Herrhausen-Straße 50 58448 Witten .

E-mail: joergf@uni-wh.de

Bibliografie

Suchttherapie 2005; 6: 60-65 ๑ Georg Thieme Verlag KG Stuttgart · New York

DOI 10.1055/s-2005-858342

ISSN 1439-9903 
änderungen, Muskelspannung, Hautwiderstand und Atemtiefe deutlich wurde, sondern auch der Blutfluss in Hirnstrukturen, die nach gegenwärtiger Erkenntnis auch bei der Verarbeitung emotionaler Reize beteiligt sind. Das Muster der Aktivierung (Blutfluss) von Regionen im Gehirn (Zunahme: ventrales Striatum, dorsomediales Mittelhirn, Insula, orbitofrontaler Kortex; Abnahme: Amygdala, linker Hippocampus, ventromedialer präfrontaler Kortex) zeigte eine verblüffende Ähnlichkeit mit Aktivitätsmustern, die von Drogen hervorgerufen werden, die, wie beispielsweise Kokain, eine primär euphorisierende Wirkung haben, was darauf hindeutet, dass die Wahrnehmung von geliebter Musik direkt mit Strukturen interagiert, die mit Emotionen assoziiert sind [1].

\section{Drogenrituale und Kultur}

Walter Freeman [2] diskutiert, wie Musik und Tanz mit der kulturellen Evolution des menschlichen Verhaltens und Bindungsformen in Beziehung standen. Er sieht einen Zusammenhang in dem tradierten Wissen über veränderte Wachbewusstseinszustände, ausgelöst durch chemische und verhaltenstechnische Induktionsformen. Die so erreichten Trancezustände sorgten einerseits für eine gezielte Durchbrechung von erlernten Gewohnheiten und Glaubenssystemen über die Wirklichkeit, aber andererseits auch für eine erhöhte Aufnahmebereitschaft neuer Informationen. Solche gezielten Veränderungen führten möglicherweise zu Formationen von „eingeweihten” Gruppen und Vertrauen bei der Weitergabe von wichtigen Erkenntnissen. Insbesondere musikalische Fähigkeiten schienen für die effiziente Tradierung von Wissensbeständen von Bedeutung zu sein.

Taeger [3] untersuchte interpersonelle Zusammenhänge von Psychedelika und religiös-mystischen Aspekten in der Gegenkultur der 70er-Jahre. Er fand viele Hinweise auf spirituelle Erfahrungsmuster und Haltungen von Musikern und Künstlern auf den LP-Hüllen und in Texten von Popkünstlern der 60er- und 70er-Jahre. Psychedelika boten einen Zugang zum kollektiven Unterbewussten der Menschheit. Viele Bilder und Symbole von Textinhalten zeugten von einer durch Psychedelika hervorgerufenen mystischen Erfahrung, wie sie schon von C. G. Jung in seiner Archetypenlehre beschrieben worden war [3]. Sheila Whiteley analysierte Musikstücke von Pink Floyd, den Beatles und anderen Musikgruppen der 60er- und 70er-Jahre und entwickelte das Konzept des „Psychedelic Coding“ [4], das symbolische und semiotische Kodierungen von Inhalten der "psychedelischen Kultur" in der Komposition beschreibt. Durch die Analyse von Text und musikalischem Material diskutiert sie Verbindungen von kultureller Semantik und Drogenwirkungen in Musik und soziokulturellem Kontext der analysierten Bands, die sich in der Produktion eines bestimmten Sounds ([5], vgl. Whiteley in [6]) von anderen Bands unterschieden. Nach Böhm [7] sind Sound, Improvisation und Ekstase stilbildende Elemente des Psychedelic Rock. Er untersuchte mögliche Wirkungen von Psychedelika auf den kompositorischen Prozess am Beispiel der BeatlesSchallplatte „Sgt. Pepper's Lonely Hearts Club Band“. Durch gezielten Einsatz von Klangmodulatoren und Studiotechnik lässt sich eine „Musik produzieren, wie sie eine Person unter dem Einfluss von psychedelischen Drogen hören möchte“ [7].
Paul McCartney antwortete auf die Frage, was die Musik der Beatles in der Zeit der Veröffentlichung des Albums „Sgt. Pepper's Lonely Hearts Club Band“ inspiriert hätte oder zum Ausdruck bringen wolle: „Vor allem Drogenerfahrungen. Aber versuchen Sie sich zu erinnern, unser Drogenkonsum stand 1967 in einer langen Musikertradition. Wir wussten von Louis Armstrong, Duke Ellington und Count Basie, das sie zeitlebens gekifft hatten. Nun war unsere Musikerszene dran, ihre Erfahrungen zu machen. Drogen fanden ihren Weg in alles, was wir taten. Sie färbten unsere Sicht der Dinge bunt. Ich glaube, dass wir damals realisierten, dass es weniger Grenzen für uns gab als wir angenommen hatten. Und uns wurde klar, dass wir Barrieren durchbrechen konnten" [8].

Jeder populäre Musikstil ist zugleich Ausdruck eines Lebensstils und entsprechender Konsumvorlieben der die Musikstile prägenden Künstler und Künstlerszenen [4, 9]. So ist die Vorliebe einer kulturellen „Szene” für bestimmte Drogen immer auch eine Mode, sich „anzutörnen“, d.h. sich in bestimmte physiologische Zustände zu versetzen, um Alltägliches und Besonderes, Ereignisse und Stimmungen intensiver und aus einer anderen Perspektive zu erfahren und dies kreativ zu nutzen.

Barbara Kerr [10] fand in einer Befragung zu Konsumgewohnheiten unter Künstlern $(n=86)$ eine signifikant höhere Konsumbereitschaft für Cannabis unter Musikern als bei anderen Künstlern. Baumeister [11] reflektiert den Acid-Rock der 60er- und 70er-Jahre, Reggae-Musik und Kultur der Rastafaris in Jamaika untersuchten Blätter und Rubin [vgl. 9, 12], und Behr berichtet über die durch Cannabis inspirierte Rembetiko-Musik im Griechenland der 30er-Jahre [vgl. 13]. Entwicklungen im Punk, Grunge, Hip-Hop, Metal, Techno, Goa-Trance etc. [14-16] können hier nur kurz erwähnt werden. Alkohol, Tabak, psychotrope Medikamente und Kaffee als Vertreter der legalen Drogen halten sich als Begleiter der Musiker bis in die heutige Zeit [17]. Berlioz' „Symphonie Phantastique ", in der dem Programmheft nach auch Opiumerfahrungen vertont werden, und Wagners musikalische Beschreibungen der „bewusstseinsverändernden Qualitäten von Isoldens Liebestrank“ [18] lassen erahnen, dass die derzeit üblichen opiumhaltigen Genussdrogen auch den Komponisten nicht unbekannt waren.

\section{Rave-Parties}

Mit der Entwicklung des Techno, der - wie Philip Tagg es formulierte - den rhythmischen Hintergrund der Musik in den Vordergrund geholt hat, ging eine Partykultur einher, die zu maschinengenerierten Grooves mit bis zu 300 bpm in nächtelangen Tanzparties („Raves”) moderne Trancerituale inszenierte. Wie schon Rouget [19] zeigte, sind verschiedene Tanzformen während institutionalisierter oder privater Trancerituale aus fast allen Kulturen bekannt. Trancemusik und hier auch Techno zeichnet sich „durch eine allmähliche steigernde Intensität aus. Akzelerierendes Tempo, Zunahme der Lautstärke, zusätzliche polyrhythmische Verschiebungen oder ein intensivierter neuer Einsatz der Musik nach einer abrupten Pause führen die Zuhörer und Tänzer oft zur tranceauslösenden ,Krise'“ [20]. Die 21 Interviewpartner in Mitterlehners Studie berichteten übereinstimmend von einer „Schwelle“, die überwunden werden muss. 
Während Musik und ihre manipulierte Lautstärke, Tanz und visuelle Stimulation durch Licht, Farben, etc. die psychophysiologischen Veränderungen - intensiviert noch durch die synthetische Droge Ecstasy (MDMA) - hervorrufen, bereitet der symbolische Rahmen der Rave-Party das „technoschamanische” Setting für veränderte Wachbewusstseinszustände. Hutson [16] widmete sich in einer Cyber-Ethnographie von Internetforen den Beschreibungen von spirituellen Erfahrungen und veränderten Wachbewusstseinszuständen. Partys im Freien oder an besonderen Orten werden von den Besuchern bevorzugt. Dabei wird die „Rave-Party” eher als ein modernes Stammesritual (vgl. [15]) verstanden, auf dem die „Hohenpriester” an den Samplern und Plattentellern stehen, die Adepten des Tanzkults ihr "Sakrament” in Form einer illegalen synthetischen Droge einnehmen und als Höhepunkt ihrer Kultur und ihres gemeinschaftlichen Ethos eine Million Menschen auf einer ehemals von Militär und Diktatur symbolisierten Strasse tanzen lassen [21]. Einig scheinen sich die Tanzenden in Mitterlehners und Hutsons Befragung jedoch darin zu sein, dass es auch ohne Drogen zu veränderten Wachbewusstseinszuständen kommt, die Musik, der Tanz und die Party sich allerdings mit der Droge noch intensiver erleben ließen.

Schon in den 40er-Jahren konnte festgestellt werden (Walter in [16]), dass rhythmische Lichtemissionen („photic driving”) visuelle Imaginationen von Bewegung, Mustern und Farben auslösen. Massenphänomene, Lichtinszenierungen und Einzelstimuli wie Dehydration ergeben während einer Techno-Party eine individuell erlebte Reizüberflutung. Tanzen bis zur Erschöpfung, die extreme physische Anstrengung und eine dadurch vermittelte Ausschüttung von körpereigenen Morphinen, die wiederum angstlösend und euphorisierend wirken, des weiteren Schwitzen und - durch die lange Dauer solcher Veranstaltungen - Schlafentzug aktivieren körpereigene Drogen und Belohnungssysteme, d.h. eine Trance stellt sich nicht einzig aus der Einnahme von Drogen ein. Ebenso wenig ist zu erwarten, dass die Musik und hier insbesondere der Rhythmus allein einen veränderten Wachbewusstseinszustand auslösen.

\section{Musikwahrnehmung}

Cannabis wirkt im Rauschverlauf unterschiedlich anregend, psychedelisch oder beruhigend. Das hängt von Dosis und Erfahrung $\mathrm{ab}$ und in welcher Stimmung und Umwelt sich der Berauschte befindet [22, 23]. Bei hohen Dosierungen kann es zu Synästhesieerfahrungen kommen: „Die Töne bekleiden sich mit Farben und die Farben enthalten Musik“ [22].
Curry [24] befragte und beobachtete während der 60er-Jahre drogenkonsumierende Musiker und Hörer in Clubs und auf Konzerten. Drogenwirkungen von Cannabis, Psychedelika und Amphetaminen auf die Musikwahrnehmung interpretierte er als eine Veränderung des kognitiven Stils, i. S. e. Hyperfokussierung der Wahrnehmung auf den Klang und eine innere Reise in den akustischen Raum. „(...) Eine Reorientierung der Wahrnehmung findet statt, der begrenzte visuelle Raum verschwindet und der als sphärisch erfahrene akustische Raum wird erlebt und darauf reagiert" [24]. Dieser Aspekt einer Hyperfokussierung der Wahrnehmung auf den akustischen Raum und eine durch synästhetische Muster geprägte innere Reise in den Klang zeigten sich auch in den Untersuchungsergebnissen von Charles Tart. Er lies 151 Cannabiskonsumenten folgende Statements auf einer Ratingskala beurteilen [25] (vgl. Abb. 1).

Von Aldrich [26] und Reed [27] wurden leichte Verbesserungen beim Seashore-Rhythm-Test nachgewiesen. Melges et al. erklärten die Auswirkung auf die Zeitwahrnehmung als eine reziproke Beziehung von subjektiv verlangsamter Zeit, i. S. e. Zeitdehnung und einer cannabisinduzierten Beschleunigung der „inneren“ Uhr $[28,29]$. In audiologischen Tests veränderte Cannabis die auditorische (Intensitäts-)Metrik von Versuchspersonen [30] und induzierte Frequenzpräferenzen zugunsten höherer Frequenzen [31]. Beschreibungen synästhetischer Effekte bei Baudelaire und Tart, geschwächte Zensur visueller Tiefenwahrnehmung in Emrichs Untersuchungen [32], kreativere Rohrschachmuster-Interpretationen und ein Übergang zu divergentem Denkstil deuten auf eine Intensivierung der individuellen zerebralen Hörstrategie i. S. e. Hyper-Fokussierung der Wahrnehmung auf den akustischen Raum-Sound und die (Zeit-)Struktur der Musik. Aus den genannten Untersuchungen (in [33,34]) lässt sich demnach schließen, dass Cannabis weniger Wirkungen auf die Musikwahrnehmung „per se” hat, sondern durch die Veränderung von Zeit-, Frequenzund Raumwahrnehmung, ähnlich einem Enhancer, Exciter oder Kompressor aus der Studiotechnik, auf die Fokussierung der Wahrnehmung von raumzeitlichen Klanggestalten wirkt und sich dadurch vermittelt, als eine psychoakustische Qualitätssteigerung erleben lässt. Den Mix des neu aufgenommenen Stücks noch einmal unter Cannabiseinfluss anzuhören, gehört bei verschiedenen Musikern anscheinend zur Begutachtungsstrategie, wie Aussagen von Mitgliedern der Beatles oder Fleetwood Mac bestätigen [35]. Der musikalisch-akustische Zeit-Raum der Klänge, ihr „Sound Staging", lässt sich mit dem durch Drogen veränderten metrischen Bezugsrahmen hörend, komponierend und improvisierend gestalten - wenn man damit erfahren ist [33].

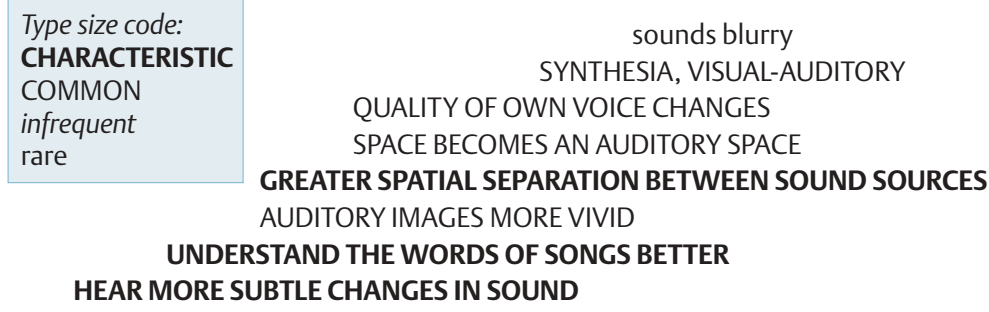

\section{D}

just fairly strongly very strongly maximum
Abb. 1 Dosis und Wirkungszusammenhänge von Cannabis und Musik nach Tart [25]. 
EEG-Studien von Hess und Fachner zeigen, dass der Vorgang des Hörens temporär intensiviert und fokussiert ist und sich die individuelle Hörstrategie ändert. Hess [36] untersuchte die Wirkungen von Cannabis und ihre Korrelationen im EEG bei Flimmerlicht, Musik und einer Hyperventilationsphase. Er fand frontale und parietale Zunahmen von Alpha und ein Absinken der Frequenz in Korrelation zum Kontemplationsstadium der Cannabiswirkungen. Beim Hören der Musik zeigten sich die „deutlichsten Zeichen des Haschischrauschs“ [36], und der veränderte Wachbewusstseinszustand ließ sich durch die Musik steuern. Die Musik wurde als intensiviert empfunden, Details besser wahrgenommen, und das Zeitempfinden beim Hören der Musik veränderte sich deutlich.

Fachner [34] fand im EEG beim Musikhören ohne Cannabis stärkere, mit Cannabis hingegen schwächere Amplituden und Frequenzmengen über nahezu alle Gehirnareale im Vergleich zur Ruhe. Doch im Parietallappen, der Aufmerksamkeit und Wahrnehmung koordiniert, zeigte sich beim Hören nach Cannabis ein deutlicher Anstieg der Alpha-Amplituden. Alphazunahme im umgekehrten Verhältnis zur kognitiven Arbeit („reverse Alpha”) wird in der EEG-Literatur als ein Hinweis auf eine leichtere mentale Verarbeitung diskutiert (vgl. [34]).

Thetaveränderungen im rechten Schläfenlappen (temporal) und auf dem Alphaband im linken Hinterhauptlappen (okzipital) waren signifikant $(\mathrm{p}<$.025). Okzipitale Veränderungen deuten auf akustische Wahrnehmungsveränderungen, temporale auf Änderungen im auditorischen System, Mittelhirn und dem limbischen System. In den Regionen des Mittel- und Kleinhirns, die vornehmlich Intensitätsempfindungen, Gedächtnis-, Selektions-, Zeit- und Bewegungsprozesse verarbeiten, gibt es proportional stärkere Ansammlungen von Cannabinoidrezeptoren. Durch Cannabis angeregt, erzeugen diese Rezeptoren eine im topographischen EEG nachvollziehbare Bahnung und Hemmung kortikaler Prozesse i. S. e. Fokussierung der Aufmerksamkeit. Funktional äußert sich dies in einem temporär veränderten, möglicherweise effizienteren metrischen Bezugsrahmen von Intensität, Akustik und Rhythmus [33, 34].

Harrer konnte durch mehrere Einzelbeobachtungen eine dosisabhängige Beeinflussung des Musikerlebens durch Benzodiazepine beschreiben. Dabei wurden die normalerweise variablen vegetativen Reaktionen auf Musik, wie Atmung, Herzschlag etc. immer deutlicher eingeschränkt, ohne dass es zu einer für die Versuchspersonen erkennbaren Beeinträchtigung oder Änderung ihres Musikerlebens kommt. Bei mittlerer Dosis waren keine psychischen Beeinträchtigungen, aber eine Unterdrückung der sonst auftretenden, typischen vegetativen Abläufe zu beobachten. Eine hohe Dosis transformierte das emotionale Erleben in ein ästhetisch-wertendes Musikerleben und variable Abläufe des Vegetativums blieben fast völlig aus. Bei sehr hohen Dosen Benzodiazepin zeigte sich die Person der Musik gegenüber gleichgültig und desinteressiert, stumpfte affektiv ab und befand sich in einer vegetativen Starre [37].

\section{Therapeutische Interventionen}

Die rezeptive Musiktherapie des Guided Imagery in Music entstand aus der psychedelischen Therapie, in der unter psychothe- rapeutischer Begleitung LSD, Meskalin oder Psilocybin genommen wurden. Dies geschah, um in therapeutischer Absicht die Abwehr- und Selektionsmechanismen der Psyche zu schwächen und um somit einen ungebremsten Assoziationsfluss für die Themen der Psychotherapie hervorzubringen [6, 38-40]. De Rios [41] fordert für die therapeutische Praxis mit Drogen und Musik einen musikwissenschaftlichen Ansatz, um therapeutisch intendierte veränderte Wachbewusstseinszustände induzieren und durch Musik führen zu können. De Rios' ethnologische Feldforschungen und Analysen unterstreichen, dass die therapeutische Praxis von Beobachtungen solcher kultureller Traditionen lernen und ein Transfer möglich sein könnte.

\section{Psilocybin}

Die psychedelische Droge Psilocybin wurde von Weber [42] eingesetzt, um modellhaft („Modellpsychose”) die Wahrnehmungswelt von Menschen zu verstehen, die durch einen psychotischen Schub eine lebhafte und manifeste Phantasie entwickeln. Weber ging dabei davon aus, dass sich die Musikwahrnehmung eines erwachsenen Menschen im Sinne einer funktionalen Regression auf kindliche Wahrnehmungs- und Kognitionsformen zurückbewegt. Die veränderte Wahrnehmung von Zeit und Körperschema fand ihre Entsprechung in einer Alteration der in der Musik gehörten Form, Bewegung und Gestalt. Angelehnt an Piagets Entwicklungsmodell wurden die Erfahrungen als primär emotional, synkretistisch und egozentrisch interpretiert. Zustandsspezifische Prozesse lassen sich i.S.d. Modellpsychose vergleichend beobachten. Zustandsspezifische Erinnerungen haben in der Musiktherapie mit Dementen und Alzheimerpatienten Bedeutung, da sie durch das Singen von Liedern ausgelöst werden können und durch intensivere Erinnerungen an Vergangenes zu einer in der Musik strukturierten Gegenwart verhelfen. Drogenabhängige in der Musiktherapie scheinen hingegen durch Musik an Zustände der Drogenwirkung erinnert zu werden [43].

\section{Ayahuasca}

De Rios [41] studierte in den 60er- und 70er-Jahren die Rolle von pflanzlichen Halluzinogenen und Musik, die Schamanen als Unterstützung ihrer Heilungsrituale nutzen. Mit dem Musikwissenschaftler Fred Katz transkribierte De Rios Musik aus therapeutischen Sitzungen mit Ayahusaca, die bei Mestizen in Iquito, Peru, aufgenommen wurde. Die Heiler betonen die Wichtigkeit der Musik und insbesondere der Melodien („Icaros”), mit denen sie den Inhalt ihrer durch Ayahuasca begünstigten Visionen programmatisch steuern, Geister oder Ahnen anrufen können, um den Grund der Erkrankung ihrer Patienten zu erkennen.

De Rios und Katz interpretieren die Funktion der Musik als ein Gerüst (,jungle gym“), das Teilnehmern in Drogenritualen einen definierten Rahmen bietet, innerhalb dessen sie begleitet ihre „Reise" ins Unterbewusste führen können [in 41]. Dabei dienen die Musik und ihre innere Struktur, um in drogenbedingten Phasen der Ich-Auflösung einen funktionalen Ersatz für die psychische Struktur zu bieten, also nicht nur dafür, innerhalb eines bestimmten Settings für Stimmung zu sorgen. Die Musik ermöglicht den Teilnehmern durch Gesänge, Pfeifen oder Trommelschläge den „leitenden Geist des Ayahuasca“ zu erkennen oder den Kontakt zu einer speziellen, für das Ritual wichtigen, Gottheit zu erreichen. Entsprechend sollten sich die Leiter solcher Rituale auch mit der Musik, den Substanzen, den ritualimmanenten Zielen und den 
bei den Teilnehmern hervorgerufenen strukturierten Visionen auskennen. Die zum Einsatz kommende Musik dient dabei kulturellen Zielen und evoziert stereotype Visionen, die - wie De Rios in einer kulturvergleichenden Untersuchung von Halluzinogen herausfand - in der jeweiligen Kultur eine ganz bestimmte Bedeutung haben. Synästhesie scheint für Rituale mit Drogen ein wichtiger Bestandteil zu sein, insofern als Halluzinogene die sensorischen Überkreuzungen der Sinnesmodalitäten von Geruch, Sehen, Fühlen, Tasten, Hören und Schmecken zu einer erhöhten Übereinstimmung von Inhalt und Form führen und Visionen lebendig machen [41].

\section{Iboga}

Maas und Strubelt studierten im afrikanischen Gabun die Musik und Pharmakotherapie traditioneller Heiler. Während der Zeremonien wird die psychedelisch wirksame pflanzliche Droge Iboga genommen, die primär auf die Koordination des Kleinhirns wirkt. Der Mediziner und Musiker Maas begab sich in ein Initiationsritual und berichtet von den erfahrenen und beobachtbaren Vorgängen, beschreibt den Gebrauch der Instrumente und analysiert anhand der rhythmischen Muster die funktionale Bedeutung der Musik.

In der Trance-Induktionsphase überlagerten schnelle Rhythmen funktionell ein zuvor durchgängiges, komplexes Muster aus walzer- und marschähnlichen Strukturen. Die rituelle Bedeutung der Polyrhythmen wird von dem traditionellen Heiler Lubin als „there are always various paths and multiple crossroads“ erklärt [44]. Die Autoren diskutieren, dass Musik und Instrumente nicht nur eine kulturelle Funktion, sondern in Verbindung mit der Droge einen direkten Einfluss auf somatische Funktionen haben. Das konstante Metrum, die überlagernden Polyrhythmen, die Harmonien und die Wahl der Instrumente dienen offensichtlich einer auf die Drogenwirkung und den Verlauf des Rituals abgestimmten Aktivierung des Kleinhirns, die die Musik als auch die Wirkungen der Droge und die hervorgerufenen spezifischen Visionen gezielt unterstützt. Während des dreitägigen Rituals bleibt die polyrhythmische Musik beständig vorhanden und nach den Erfahrungen der Autoren stellt sich ein anhaltendes „inneres Metrum” ein, das sie als mathematisch analog einer $6 \mathrm{~Hz}$-Frequenz des Thetabands im EEG interpretieren.

\section{Literatur}

${ }^{1}$ Blood AJ, Zatorre RJ. Intensely pleasurable responses to music correlate with activity in brain regions implicated in reward and emotion. Proc Natl Acad Sci USA 2001; 98: 11818-11823

2 Freeman W. A neurobiological role of music in social bonding. In: Wallin NL, Merker B, Brown S (eds). The origins of music. Cambridge, Mass: MIT Press, 2000: $411-424$

${ }^{3}$ Taeger HH. Spiritualität und Drogen. Interpersonelle Zusammenhänge von Psychedelika und religiös-mystischen Aspekten in der Gegenkultur der 70er-Jahre. Markt Erlbach: Raymond Martin, 1988

${ }^{4}$ Whiteley S. The space between the notes: rock and the counter culture. London: Routledge, 1992

${ }^{5}$ Whiteley S. Altered Sounds. In: Melechi A (ed). Psychedelia Britannica. London: Turnaround, 1997: 120-142

${ }^{6}$ Melechi A. Psychedelia Britannica. London: Turnaround, 1997

${ }^{7}$ Böhm T. Was ist Psychedelic Rock? Zum Einfluss von Drogen auf die Musik am Beispiel der Beatles und LSD. In: Rösing H, Phleps T (Hrsg). Erkenntniszuwachs durch Analyse. Populäre Musik auf dem Prüfstand. Karben: Coda, 1999: 7-25
${ }^{8}$ Davis A, Pieper W. Die psychedelischen Beatles. Löhrbach: Werner Piepers MedienXperimente, 1993

${ }^{9}$ Shapiro H. Sky High. Drogenkultur im Musikbuisiness. St. Andrä-Wördern: Hannibal, 1998

${ }^{10}$ Kerr B. Substance abuse of creativly talented adults. Journal of Creative Behaviour 1992; 25: 145 - 153

${ }^{11}$ Baumeister RF. Acid rock: a critical reappraisal and psychological commentary. J Psychoactive Drugs 1984; 16: 339-345

12 Blätter A. Kulturelle Ausprägungen und die Funktion des Drogengebrauchs. Hamburg: Wayasbah Verlag, 1990

${ }^{13}$ Behr HG. Von Hanf ist die Rede. Basel: Sphinx Verlag, 1982

${ }^{14}$ Lyttle T, Montagne M. Drugs, music, and ideology: a social pharmacological interpretation of the Acid House Movement. Int J Addict 1992; 27: $1159-1177$

15 Rätsch C. Ritueller Gebrauch psychoaktiver Substanzen im modernen Mitteleuropa. Curare 1995; 18: 297-324

${ }^{16}$ Hutson SR. The rave: spiritual healing in modern western subcultures. Anthropological Quarterly 2000; 73: 35-49

17 Bessler J, Opgenoorth N. Anekdoten aus der Musikwelt. Bonn: Voggenreither, 2000

18 Rätsch C. Musique Fantastique. In: Höhle S, Müller-Ebeling C, Rätsch C et al (Hrsg). Rausch und Erkenntnis. Das Wilde in der Kultur. München: Knaur-Verlag, 1986: 307-325

${ }^{19}$ Rouget G. Music and trance: a theory of the relations between music and possession. Chicago: University Press, 1985

${ }^{20}$ Mitterlehner F. Let's fly together. Zur Untersuchung veränderter Bewusstseinszustände während einer Techno-Party. In: Leuner HC, Schlichting M (Hrsg). Jahrbuch des Europäischen Collegiums für Bewusstseinsstudien. Berlin: Verlag für Wissenschaft und Bildung, 1996: 49-62

${ }^{21}$ Saunders N. Ecstasy and the dance culture. London: Turnaround, 1995

22 Baudelaire C. Die künstlichen Paradiese. Die Dichtung vom Haschisch. Zürich: Manesse Verlag, 1988

${ }^{23}$ Julien RM. Drogen und Psychopharmaka. Heidelberg: Spektrum Akademischer Verlag, 1997

${ }^{24}$ Curry A. Drugs in rock and jazz music. Clinical Toxicology 1968; 1 : $235-244$

25 Tart C. On being stoned: a psychological study of marihuana intoxikation. Palo Alto: Science and Behaviour Books, 1971

${ }^{26}$ Aldrich CK. The effect of synthetic marihuana-like compound on musical talent. Public Health Report 1944; 59: 431-435

${ }^{27}$ Reed HBC. Cognitive effects of marihuana. In: Mendelson JH, Rossi AM, Meyer RE (eds). The use of marihuana: a psychological and physiological inquiry. New York: Plenum Press, 1974: 107-114

${ }^{28}$ Melges FT, Tinklenberg JR, Hollister LE et al. Marihuana and temporal disintegration. Science 1970; 168: $1118-1120$

${ }^{29}$ Melges FT, Tinklenberg JR, Hollister LE et al. Marihuana and the temporal span of awareness. Arch Gen Psychiatry 1971; 24: 564-567

${ }^{30}$ Globus GG, Cohen HB, Kramer JC et al. Effects of marihuana induced altered state of consciousness on auditory perception. Journal of Psychedelic Drugs 1978; 10: $71-76$

${ }^{31}$ De Souza MR, Karniol IG, Ventura DF. Human tonal preferences as a function of frequency under delta8-tetrahydrocannabinol. Pharmacol Biochem Behav 1974; 2: 607-611

32 Emrich HM. Psychiatrische Anthropologie. Therapeutische Bedeutung von Phantasiesystemen. München: Pfeiffer, 1990

${ }^{33}$ Fachner J. Cannabis, Musik und ein veränderter metrischer Bezugsrahmen. In: Rösing H, Phleps T (Hrsg). Populäre Musik im kulturwissenschaftlichen Diskurs. Beiträge zur Popularmusikforschung. Karben: Coda, 2000: 107-122

${ }^{34}$ Fachner J. Topographic EEG changes accompanying cannabis-induced alteration of music perception: cannabis as a hearing aid? Journal of Cannabis Therapeutics 2002; 2: 3-36

35 Boyd J. Musicians in tune. New York: Fireside, 1992

${ }^{36}$ Hess P. Licht und Schatten. Hanf aus medizinisch-psychiatrischer Sicht. In: Cosack R, Wenzel R (Hrsg). Das Hanf-Tage-Buch. Neue Beiträge zur Diskussion über Hanf, Cannabis, Marihuana. Hamburg: Wendepunkt, 1995: 25-44

${ }^{37}$ Harrer G. Zur Bedeutung psychischer und somatischer Reaktionen beim Erleben von Musik. In: Rösing H (Hrsg). Musik als Droge? Zu Theorie und Praxis bewusstseinsverändernder Wirkungen von Musik. Mainz: Villa Musica, 1991: 9-21

${ }^{38}$ Eagle CT. Music and LSD: an empirical study. Journal of Music Therapy 1972; 9: $23-36$ 
${ }^{39}$ Bonny HL, Pahnke WN. The use of music in psychedelic (LSD) psychotherapy. Journal of Music Therapy 1972; 9: 64-87

${ }^{40}$ Grof S. Topographie des Unbewussten. Stuttgart: Klett-Cotta, 1993

${ }^{41}$ De Rios MD. The role of music in healing with hallucinogens: tribal and western studies. Music Therapy Today 2003; 4 (www. musictherapytoday.com)

42 Weber K. Veränderungen des Musikerlebens in der experimentellen Psychose (Psylocibin) und ihre Bedeutung für die Musikpsychologie. In: Revers WJ, Harrer G, Simon WCM (Hrsg). Neue Wege der Musiktherapie. Düsseldorf/Wien: Econ Verlag, 1974: 201 -225
${ }^{43}$ Horesh T. Dangerous music: working with the destructive and healing powers of popular music in the treatment of substance abusers. Music Therapy Today 2003; 4 (www.musictherapytoday.com)

${ }^{44}$ Maas U, Strubelt S. Music in the iboga initiation ceremony in Gabon: polyrhythms supporting a pharmacotherapy. Music Therapy Today 2003; 4 (www.musictherapytoday.com) 